\title{
Acquisition Guidelines and Quality Assessment Tools for Analyzing Neonatal Diffusion Tensor MRI Data
}

\author{
A.M. Heemskerk, A. Leemans, A. Plaisier, K. Pieterman, M.H. Lequin, and J. Dudink
}

\begin{abstract}
SUMMARY: Diffusion tensor imaging is a valuable measure in clinical settings to assess diagnosis and prognosis of neonatal brain development. However, obtaining reliable images is not straightforward because of the tissue characteristics of the neonatal brain and the high likelihood of motion artifacts. In this review, we present guidelines on how to acquire DTI data of the neonatal brain and recommend high-quality data acquisition and processing as an essential means to obtain accurate and robust parametric maps. Sudden head movements are problematic for DTI in neonates, and these may lead to incorrect values. We describe strategies to minimize the corrupting effects both in terms of acquisition (eg, more gradient directions) and postprocessing (eg, tensor estimation methods). In addition, tools are described that can help assess whether a dataset is of sufficient quality for further assessment.
\end{abstract}

ABBREVIATIONS: $M D=$ mean diffusivity; FA = fractional anisotropy; RESTORE = robust estimation of tensors by outlier rejection; TBSS = tract-based spatial statistics

B oth premature birth and complications around term birth are risk factors for brain injury and subsequent neurodevelopmental impairment. However, this injury often remains "silent" long after the threshold of irreversible injury has been crossed. The most important challenge is to define early proxy measurements of long-term neurodevelopmental outcome that will enable intervention in the early stages of the still adaptive developing human brain. MR imaging is widely used to monitor development and injury of the neonate brain and to predict neurodevelopmental outcome ${ }^{1-6}$ (Fig 1). Advanced quantitative MR imaging techniques such as DTI $^{7-11}$ can detect subtle differences between normal and abnormal tissue. DTI has become invaluable for the assessment of brain development in preterm infants because it enables detection of white matter maturation during premyelination. ${ }^{1,3,12-14}$ Several DTI studies have revealed that abnormal white matter maturation is correlated to neuromotor and neurocognitive performances to childhood. ${ }^{15-18}$ DTI measurements can therefore provide early biomarkers to be targeted in neuroprotective intervention trials.

Received October 3, 2012; accepted after revision November 15.

From the Division of Neonatology, Department of Pediatrics (A.M.H., A.P., K.P., J.D.), and Division of Pediatric Radiology, Department of Radiology (A.M.H., A.P., M.H.L., J.D.), Erasmus Medical Center, Rotterdam, The Netherlands; and Image Sciences Institute (A.L.), University Medical Center Utrecht, Utrecht, The Netherlands.

Please address correspondence to Jeroen Dudink, MD, PhD, Division of Neonatology, Department of Pediatrics, Erasmus Medical Center, Dr Molewaterplein 60,

3015 GJ Rotterdam, Netherlands; e-mail: j.dudink@erasmusmc.nl.

http://dx.doi.org/10.3174/ajnr.A3465
Although diffusion MR imaging of the neonatal brain is gaining popularity, time constraints often impede acquisition of high quality data. The total time in the scanner should be kept to a minimum, not only for ethical reasons but also to prevent hemodynamic instability. Moreover, a longer acquisition time increases the chance of motion. The feasibility of an MR imaging study and the optimal settings for the MR imaging scan are determined by the available time in combination with MR hardware and the requirements for obtaining reliable and meaningful data.

From the information above, it may be clear that acquisition, processing, and interpretation of the images with this advanced MR imaging technique are not as straightforward as with the conventional MR imaging sequences. Recent reviews have covered the general concerns within the DTI pipeline that can severely influence the measured results and therefore the study outcome. ${ }^{11,19,20}$ Many of the specifics of diffusion MR imaging acquisition in adult brains are also applicable to imaging the neonatal brain. However, the latter presents challenges related to size, tissue composition, and motion and therefore requires specific acquisition settings. Targeted acquisition schemes and postprocessing methods are necessary to account for motion and to obtain reliable DTI parameter maps.

In this review, we present guidelines on how to acquire diffusion MR imaging data of the neonatal brain. Furthermore, we recommend high-quality data acquisition and processing as essential means to obtain accurate and robust parametric 

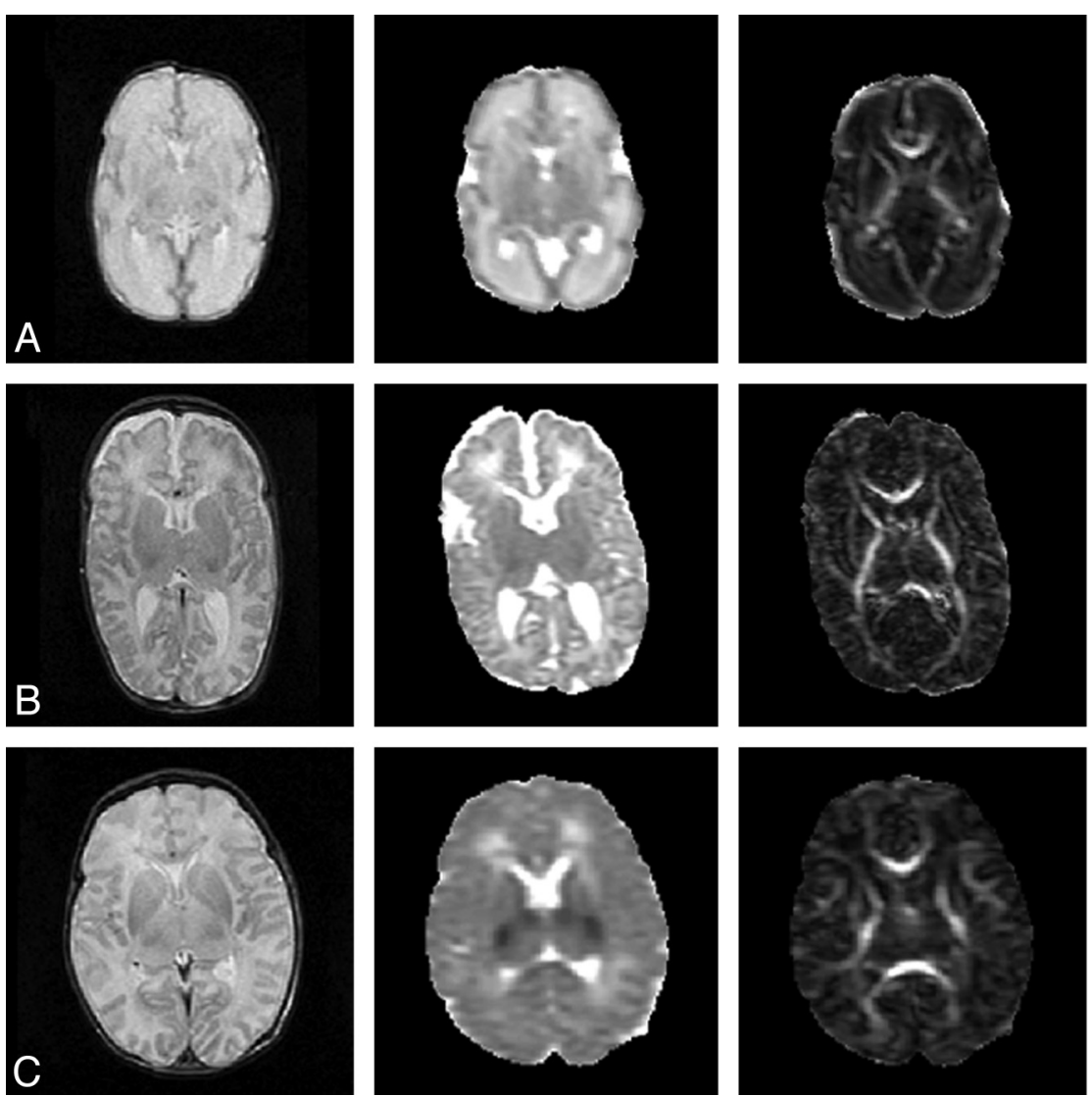

FIG 1. Anatomic, MD, and FA map of a neonate. A, At 30 weeks' gestational age; $B$, at term age; and $C$, after perinatal asphyxia scanned on day 4 , with abnormal low signal intensity in the central gray matter on the MD map. Notice the decrease in MD values and increase in FA values between the preterm and a term brain. The MD maps and FA maps are equally scaled for the 3 subjects, respectively, $0-2 \times 10^{-3} \mathrm{~mm}^{2} / \mathrm{s}$ and $0-1$.

maps. We will first describe some general topics related to DTI acquisition. Next, we broadly describe the specific concerns and acquisition settings related to DTI in the neonate. Finally, we discuss the main steps related to DTI acquisition and processing and the ways they influence the neonatal DTI data quality.

\section{DTI OF THE NEONATE}

Imaging the neonatal brain is more difficult than imaging the adult brain. The neonate head is much smaller, and the brain is developing at a fast rate, has a high water content, and is unmyelinated. ${ }^{21} \mathrm{MR}$ imaging acquisition settings therefore must be adjusted to neonatal MR imaging relaxation times and diffusion coefficients. Additionally, there is a greater likelihood of motion artifacts in the images because neonates have higher heart and breathing rates and will not lie motionless because sedation is usually not given in this situation. Fig 2 shows examples of motion artifacts. Thus, the optimal acquisition strategies established for the adult brain ${ }^{22}$ are not necessarily applicable to the neonatal brain because diffusivities, anisotropy, T2, and SNR will differ. Additionally, optimal acquisition depends on multiple, interrelated parameters, including but not limited to, TR, TE, acquisition matrix, field of view, section thickness, acquisition time, number of averages, maximum b-value, number of b-values, number of non-DWIs, and number of gradient directions.
SETUP FOR IMAGING NEONATES

Neonatal MR imaging is challenging because many neonates are receiving respiratory support and are vulnerable to hemodynamic instability. ${ }^{23-25} \mathrm{~A}$ recent study showed that despite of the use of a dedicated guideline, adverse events during neonatal MR imaging scanning were common. ${ }^{26}$ Because of the increased application of neonatal MR imaging, the provision of a safe environment during the MR imaging procedures is of great concern. An MR-compatible incubator with a specialized neonatal head coil allows the infant to be kept stable and safe during transport and MR imaging scan. A small-size neonatal head coil typically offers better SNR than the larger adult head coils. ${ }^{27}$ With improved SNR, the scan time can be reduced and/or the spatial resolution for the conventional scans can be improved.

Two common strategies are used to limit motion. One is sedation, which in neonates can compromise breathing and therefore must be performed with great caution. The second strategy is promoting sleep by placing the infant in the incubator in a comfortable and secure way. ${ }^{28}$ This will generally help, but it takes some practice to comfort the child and prevent it from waking up when imaging starts. ${ }^{29}$ The infant's perception of acquisition noise can be reduced with the use of moldable earplugs, neonatal earmuffs, and/or an acoustic hood. ${ }^{30}$

\section{RESOLUTION, TR, TE}

A striking difference between the neonatal and adult brain is the inverse contrast between white and gray matter on both the T1and T2-weighted images. ${ }^{31}$ This is caused by the high water content and the unmyelinated white matter of the neonate. T1 and T2 relaxation times quickly decrease over the first year of life, and the exact pattern is different for the different brain structures. Typical T2 relaxation times are approximately $300 \mathrm{~ms}$ at $<30$ weeks' gestational age, approximately $200 \mathrm{~ms}$ at term age, ${ }^{32}$ and approximately $100 \mathrm{~ms}$ at adulthood. ${ }^{33} \mathrm{~T} 1$ relaxation times for white matter are approximately $1600 \mathrm{~ms}$ at term age and approximately 500 ms at 2 years of age. ${ }^{31}$ For DTI measurements, a longer TR can be needed to reduce the effect of the high $\mathrm{T} 1$ relaxation time (ie, saturation and therefore reduced SNR and/or bias to other proton pools). Fortunately, the long T2 relaxation time does favor the DTI measurement because there is more signal left at a similar TE. However, the DTI estimates for different ages or brain structures must be interpreted carefully because they can be influenced by the varying $\mathrm{T} 1$ and $\mathrm{T} 2$ relaxation times, possibly caused by altered relative contributions of the different water compartments (eg, intracellular versus extracellular).

AJNR Am J Neuroradiol 34:1496-505 Aug 2013 www.ajnr.org 

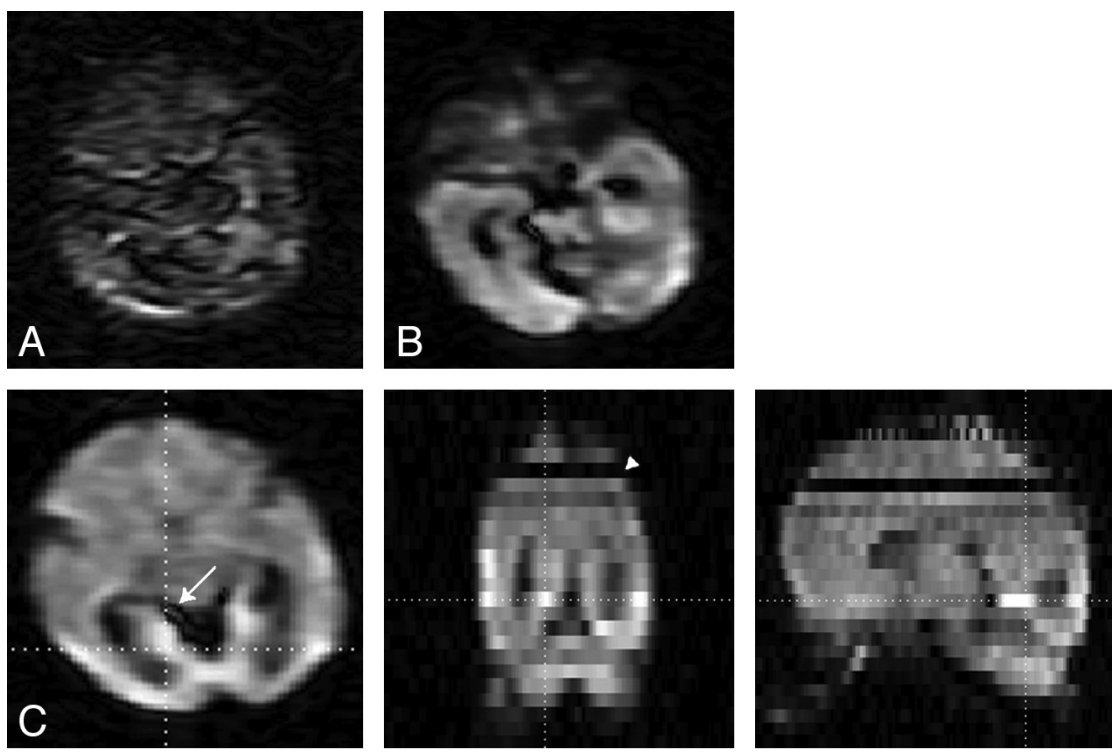

the accuracy of the diffusion estimates (eg, increased FA) and decrease precision (eg, larger standard deviations). ${ }^{37}$ The SNR dependence is influenced by the underlying diffusivities and FA values and by the acquisition settings such as b-value. ${ }^{37,38}$

The magnetic field strength affects the quality of the images both positively and negatively. At 3T, the SNR is generally higher than at $1.5 \mathrm{~T}$. However, the images acquired at $3 \mathrm{~T}$ are more sensitive to susceptibility artifacts, which are prone around air-tissue boundaries. Therefore, the field strength must be considered carefully when setting up a protocol.

The performance of the gradient sys-

FIG 2. Effects of motion and pulsation. A, Almost complete signal loss caused by head motion; $B$, signal drop-out caused by pulsation; and C, 3-plane view in which the effects of pulsation (arrow) and motion (arrowhead) are visible.

All structures in the neonatal brain are smaller than in the adult brain, thereby requiring a higher resolution. Typical inplane resolutions range from $0.6 \times 0.6 \mathrm{~mm}^{2}$ to $2 \times 2 \mathrm{~mm}^{2}$, with a section thickness of $1.9-3.0 \mathrm{~mm}$ (Table 1 ), which is still relatively large; therefore images should be interpreted cautiously. ${ }^{34}$ Additionally, the in-plane resolution is often obtained with a low acquisition matrix that is zero-filled to a higher matrix; the images therefore are smoother and boundaries are less sharp. The imaging resolution and the partial volume effects determine what structures can be resolved. For fiber-tracking purposes, the resolution is ideally isotropic and contiguous sections are needed without section gap. The use of isotropic voxels prevents preferential averaging of fiber orientation along a certain axis. ${ }^{11}$

In addition, the resolution and SNR are related because higher resolution means lower SNR. Therefore, SNR constraints can limit the resolution. Higher resolutions are obtained by decreasing the field of view or increasing the matrix size. However, both options have their limitations because the field of view must fit the whole image volume, and higher matrix size introduces artifacts and increases scan time.

\section{GENERAL DTI ACQUISITION CONSIDERATIONS}

Generally, a single-shot EPI sequence allows fast acquisition but, unfortunately, is prone to artifacts that affect the data acquisition and the processing. ${ }^{11,19,20,35}$ The most critical artifacts are image distortions caused by magnetic susceptibility effects and eddy currents. Among the solutions proposed to reduce the susceptibility artifacts are parallel imaging and B0 field correction. Eddy currents can be reduced by applying bipolar gradients and dual-echo or twice-refocused sequences. ${ }^{36}$ For a more comprehensive and detailed description of these artifacts and other problems, the authors recommend the recent review by Jones and Cercignani ${ }^{19}$ or other reviews regarding DTI. ${ }^{11,20,35}$

Diffusion MR imaging is intrinsically a low SNR technique, and the SNR can significantly influence the reliability of each tensor estimate. For example, in adult brains, low SNR levels reduce tems is an important determinant of DTI image quality. Stronger gradients allow for higher diffusion weighting within a shorter time, thereby reducing TE and improving SNR and reducing artifacts. Gradient systems with advanced eddy-current compensation are preferred because these limit image distortions.

Hardware and software of the MR imaging systems differ between manufacturers and are constantly being upgraded. Therefore, imaging protocols cannot always be exchanged between sites, and what is achievable at one scanner might not be possible at another. Additionally, even equally equipped MR imaging systems can introduce a bias. ${ }^{39}$

The coil should adequately cover the volume of interest and exhibit sufficient SNR. Preferably, a multichannel coil should be used with the option to use parallel imaging. Parallel imaging techniques can shorten the EPI readout, thereby reducing imaging artifacts and TE. ${ }^{40}$

\section{DIFFUSION WEIGHTING}

The b-value indicates the amount of diffusion weighting that is applied. The optimal b-value depends on the tensor information of interest but is generally approximately 1.09/ADC. ${ }^{22}$ For clinical adult brain DTI, a b-value of $1000 \mathrm{~s} / \mathrm{mm}^{2}$ is common practice, which is a concession between a longer optimal b-value and the need for shorter echo times to ensure enough SNR. As mentioned, the neonatal brain contains more water and is less myelineated than the adult brain. Therefore, the neonatal MD is higher than the adult MD $\left(2.0 \times 10^{-3} \mathrm{~mm}^{2} / \mathrm{s}\right.$ versus $\left.0.7 \times 10^{-3} \mathrm{~mm}^{2} / \mathrm{s}\right)$ and the optimal b-value should be lower to accurately estimate the diffusion tensor. Currently, there is no consensus on the b-value in neonates, and b-values range between $600-1100 \mathrm{~s} / \mathrm{mm}^{2}$ (Table 1). Simulation studies are needed to determine the optimal bvalue, just as has been performed for adult brains.

At least 6 diffusion gradient directions and 1 non-DWI are needed to estimate the diffusion tensor. However, more directions increase the accuracy and precision of the diffusion estimates. Tensor estimates with 30 directions are statistically rotationally invariant. ${ }^{41}$ Increasing the number of directions also 
Table 1: Published DTI acquisition settings for neonates from different groups

\begin{tabular}{|c|c|c|c|c|c|c|c|c|c|c|c|c|}
\hline PMA & FS & $\begin{array}{l}\text { Neo } \\
\text { Coil }\end{array}$ & TR & TE & FOV & Thk & $\begin{array}{c}\text { Image } \\
\text { Resolution }\end{array}$ & b-Value & $\begin{array}{l}\text { No. } \\
b=0\end{array}$ & $\begin{array}{l}\text { No. } \\
\text { Dir }\end{array}$ & Sedation & Reference \\
\hline $40-45$ & 3 & - & 7745 & 48 & 180 & 2 & 1.41 & 800 & 1 & 32 & + & van Pul et al ${ }^{67}$ \\
\hline Term & 3 & - & 8400 & 84 & 220 & 2.2 & 2.20 & 750 & $?$ & $?$ & \pm & Wintermark et al ${ }^{68}$ \\
\hline $31-41$ & 1.5 & + & 4000 & 60 & 210 & 3.0 & 0.82 & 1000 & 1 & $?$ & - & Arrigoni et $\mathrm{al}^{69}$ \\
\hline $38-41$ & 3 & - & $>3000$ & 71 & 150 & 1.9 & 1.88 & 700 & 5 & 30 & - & Oishi et al ${ }^{70}$ \\
\hline Term & 3 & & 7680 & 82 & & 2.0 & 2.00 & 1000 & 7 & 42 & - & Wang et $\mathrm{al}^{71}$ \\
\hline $37-43$ & 1.5 & & 6000 & 88 & 200 & 2.5 & 1.56 & 1000 & 1 & 15 & + & Hasegawa et a $\mathrm{l}^{72}$ \\
\hline $40-44$ & 3 & & 7465 & 54 & & 2.0 & 1.40 & 1000 & 1 & 32 & + & de Bruine et $\mathrm{al}^{73}$ \\
\hline Term & 1.5 & + & 4047 & 59 & 210 & 3.0 & 0.82 & 1000 & 1 & 6 & - & Righini et $\mathrm{al}^{74}$ \\
\hline 24-33/TEA & 1.5 & + & 7000 & 100 & & 3.0 & 1.40 & 600 & 1 & 6 & - & Bonifacio et a ${ }^{75}$ \\
\hline 24-33/TEA & 1.5 & + & 4900 & 104 & 160 & 3.0 & 1.30 & $600 / 700$ & 1 & 12 & - & Bonifacio et a ${ }^{75}$ \\
\hline Term & 3 & & 5200 & 73 & & 2.0 & 2.00 & 1000 & 1 & 6 & - & Gilmore et $\mathrm{al}^{76}$ \\
\hline $25-32$ & 1.5 & + & 9150 & 98 & 200 & 3.0 & 0.78 & 1000 & 1 & 25 & - & Dudink et $\mathrm{al}^{77}$ \\
\hline $35-42$ & 1.5 & - & 5888 & 92 & 220 & 2.3 & 2.00 & 600 & 1 & 32 & - & Liu et $\mathrm{al}^{78}$ \\
\hline $39-41$ & 1.5 & - & 7000 & 74 & 180 & 2.2 & 1.40 & 700 & 1 & 15 & + & Skiold et $\mathrm{al}^{5}$ \\
\hline Term & 1.5 & - & 8000 & 100 & 240 & 3.0 & 1.88 & 700 & 1 & 10 & - & Malik et $\mathrm{al}^{79}$ \\
\hline Term & 1.5 & - & 6000 & 106 & 230 & 2.5 & 0.90 & 1100 & 16 & 44 & - & Rose et $\mathrm{al}^{80}$ \\
\hline $38-45$ & 3 & - & 8000 & 79 & 224 & 2.0 & 1.75 & 750 & 1 & 15 & \pm & Anjari et $\mathrm{al}^{13}$ \\
\hline 30 & 1.5 & + & 11725 & 90.5 & 220 & 3.0 & 0.86 & 750 & 3 & 25 & - & Erasmus MC - Sophia \\
\hline Term & 1.5 & + & 11725 & 85.6 & 220 & 3.0 & 0.66 & 1000 & 3 & 25 & - & Erasmus MC - Sophia \\
\hline
\end{tabular}

Note:-Image resolution is all squared $(\mathrm{mm})$; b-values are $\mathrm{s} / \mathrm{mm}^{2}$.

PMA indicates postmenstrual age at image acquisition (weeks); TEA, term equivalent age; FS, field strength (T); TR, repetition time (ms); TE, echo time (ms); FOV, field of view (mm); Thk, section thickness (mm); No. Dir, number of diffusion encoding directions; +, used; and -, not used; Neo, neonatal; Erasmus MC-Sophia is the hospital where the authors are affiliated.

entails increasing the number of $b=0$ acquisitions because $1 b=$ 0 image is needed for every $8-10$ DWIs. ${ }^{22}$ The optimal distribution of the gradients is a uniform distribution along the surface of a sphere, ${ }^{22}$ which minimizes the orientation dependency of the tensor estimates. Additionally, the order of the gradients ideally should be uniformly distributed in case the acquisition is corrupted by motion or interrupted acquisition. ${ }^{42,43}$ Dubois et $\mathrm{al}^{43}$ have proposed optimal diffusion gradient orientation schemes that allow calculation of the diffusion tensor estimates with the use of partial datasets.

The higher likelihood of motion in the neonates requires a higher number of gradient directions. The relative contribution of a corrupted image is less if more gradient directions are acquired. Therefore, it may be preferable to increase the number of gradient directions at the cost of reducing TR (and thereby SNR) to keep the total scan time approximately equal. In addition, we recommend the use of extra $b=0$ images because these images also can be affected by intersection and intrasection motion, and good-quality $b=0$ images are needed to correctly calculate the tensor estimates.

\section{MOTION}

Subject-related motion can affect the resulting parameter maps. The motion can be evident between images or within a single image. During the long acquisition, adult subjects tend to slowly move their heads even if they are instructed to lay still and the head is secured by padding. ${ }^{44}$ A slow displacement and/or rotation causes a misalignment between images. This can be adjusted for by image registration. The motion within a single image is caused by cardiac pulsation or sudden large amplitude subjectrelated motion. The latter occurs rarely in adults $(1.4 \%)^{45}$ but is a severe problem in neonates. Fig 2 shows the effects of severe motion, cardiac pulsation, and the large differences in signal intensities throughout the image volume.

Cardiac pulsation causes both a local deformation of the tissue and additional signal loss. The tensor estimates are then less reliable. Because neonates have much faster heart rates than adults, the likelihood that the diffusion sensitization is obtained during a pulsation is larger, leading to a higher likelihood of artifacts. The effects of cardiac pulsation can be reduced by triggering the acquisition on the cardiac cycle; however, scan times become longer because the effective TR should be at least 5 times the T1 of the tissue. For neonates, cardiac gating seems possible only by monitoring the ECG because the delay time for the pulse wave on the pulse oximeter is too long ( $249 \mathrm{~ms}$ for adults ${ }^{46}$ ) compared with the heart beat (approximately every $350 \mathrm{~ms}$ ).

Subject motion is a major concern for neonatal imaging because neonates tend to move about even when sedated. Intrasection motion from a sudden shake of the head may corrupt the image and result in miscalculation of the DTI estimates. A typical motion-corrupted image is characterized by severe signal loss; this is caused by tissue displacement during the diffusion encoding, which also causes signal loss next to the dephasing caused by diffusion. This severe signal loss cannot be recovered, and an apparent high displacement would be calculated. Improper dealing with the motion artifacts may result in biased MD and high FA values.

Only a few studies have reported data rejection on the grounds of severe artifacts. Rejection was necessary in $15-60 \%$ of the subjects, ${ }^{15,47-49}$ and the incidence of less noticeable artifacts will be larger. In a pilot study of 27 preterm neonates, we investigated the statistical outliers of the tensor regression (see "DTI Quality and Pathology" section) and showed that data of $60 \%$ of the subjects were corrupted by motion (defined as $>10$ sections with $>30 \%$ outliers) (Table 2). ${ }^{50}$ This high occurrence of motion can have devastating results. Unfortunately, the typical tensor regression method used by MR imaging vendors and commercial processing tools is insufficient with corrupted data, and it appears that many DTI users are unaware of the motion-related problems. 
Table 2: Outliers in a small pilot study (27 preterm infants scanned at 30 weeks' gestational age)

\begin{tabular}{lccc} 
& $>\mathbf{1 0} \%$ Outliers & $>\mathbf{3 0 \%}$ Outliers & $\mathbf{> 5 0} \%$ Outliers \\
\hline No. of subjects $>10$ sections & 27 & 15 & 10 \\
No. of subjects $>20$ sections & 26 & 10 & 1 \\
Mean No. of sections with outliers & 50 & 15 & 9 \\
Range No. of sections with outliers & $17-79$ & $1-38$ & $0-24$ \\
Mean percentage of sections with outliers & 9.5 & 2.9 & 1.7 \\
Range of percentage of sections with outliers & $3.3-15.7$ & $0.2-7.0$ & $0.0-4.4$ \\
\hline
\end{tabular}
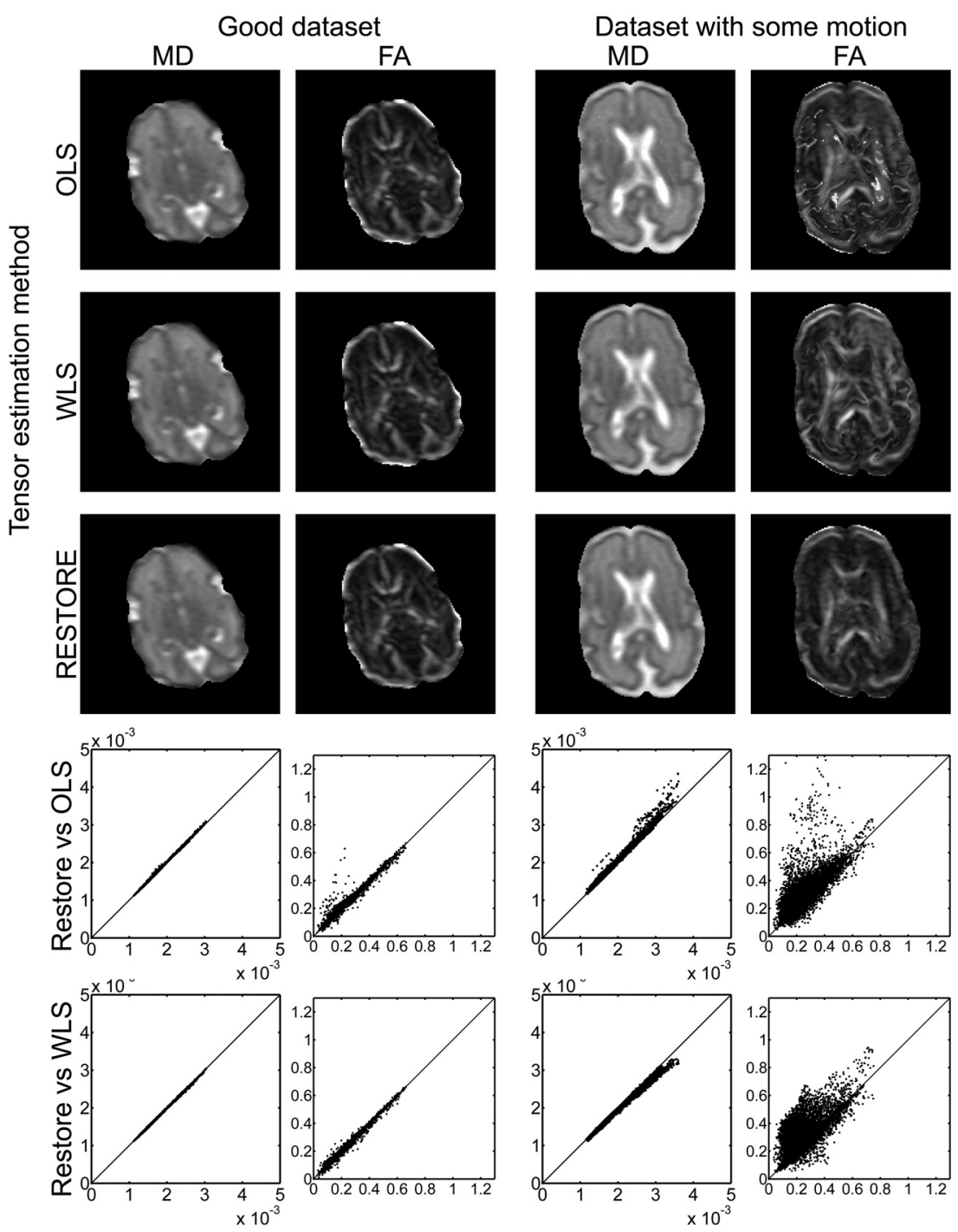

FIG 3. Effects of different tensor estimation methods. Data of 2 datasets are displayed: on the left, data without gross motion artifacts, and on the right, data with gross motion artifacts for one of the gradient directions. Both MD and FA maps are depicted, showing no visible differences for the good dataset and clearly visible differences for the dataset with motion. Especially the FA maps show that the ordinary least-squares (OLS) estimation results in very high FA values that are not related to the known anatomy. In the graphs, the pixel value of the OLS or weighted least-squares (WLS) tensor estimation is plotted against the pixel value of the RESTORE tensor estimation ( $x$-axis), and the line of identity is included. The spread around the line of identity is broader for the dataset, with motion indicating an effect of the tensor estimation method on the resulting FA or MD value. For the graphs, the images were eroded to exclude the outer rim, which contains poor-quality data caused by partial volume effects. Scaling: MD, $0-2 \times 10^{-3} \mathrm{~mm}^{2} / \mathrm{s} ; \mathrm{FA}, 0-1$.

The solutions to prevent corrupted images are limited. Obviously, the movement should be minimized a priori by placing the child comfortably and supporting the head with pads. The acquisition strategies to reduce the signal loss are limited because the the neonatal brain, in view of its different contrast. In addition, most tools perform their registration on the whole image volume and it is therefore logical that section drop-outs caused by motion severely alter the registration, especially in the case of affine reg-

sudden gross motion occurs during the time the diffusion encoding is performed. Therefore, navigator echoes or altered readout sequences appear not to be useful. However, shortening the diffusion time by stronger gradients or lower b-value might reduce the effects. Fortunately, there are strategies to decrease the detrimental effects of corrupted images. These include the oversampling of gradient directions, the removal of corrupted images, and/or the use of more advanced tensor regression methods.

\section{TEMPERATURE}

Another issue one must be aware of is that diffusion is temperature-dependent. A $1^{\circ}$ difference in temperature leads to a $2-3 \%$ difference in ADC. ${ }^{51,52}$ It will therefore be necessary to maintain the child at a constant temperature during scanning. Also, consistent core temperatures are necessary in studying group differences or performing a longitudinal study because the differences in $\mathrm{MD}$ or FA between ages, groups, and therapies are small.

\section{PROCESSING}

The basic steps in data postprocessing are motion/distortion correction, estimation of the diffusion tensor, and computation of the parametric maps. The data should be inspected before and after each step to ensure good image quality and absence of artifacts. Postprocessing results in data reduction, and it can be difficult to spot erroneous results on the parametric maps.

\section{MOTION/DISTORTION CORRECTION}

Registration and correction of the images is necessary to adjust for slight variation in brain position and to correct for the eddy current. A quick check for misregistration between images is to examine the FA maps for rims of high anisotropy at the edges of the brain or at the interface between CSF and white matter.

Because most available registration tools are optimized for the contrast in adult brain, one should check whether the registration software is adequate for 

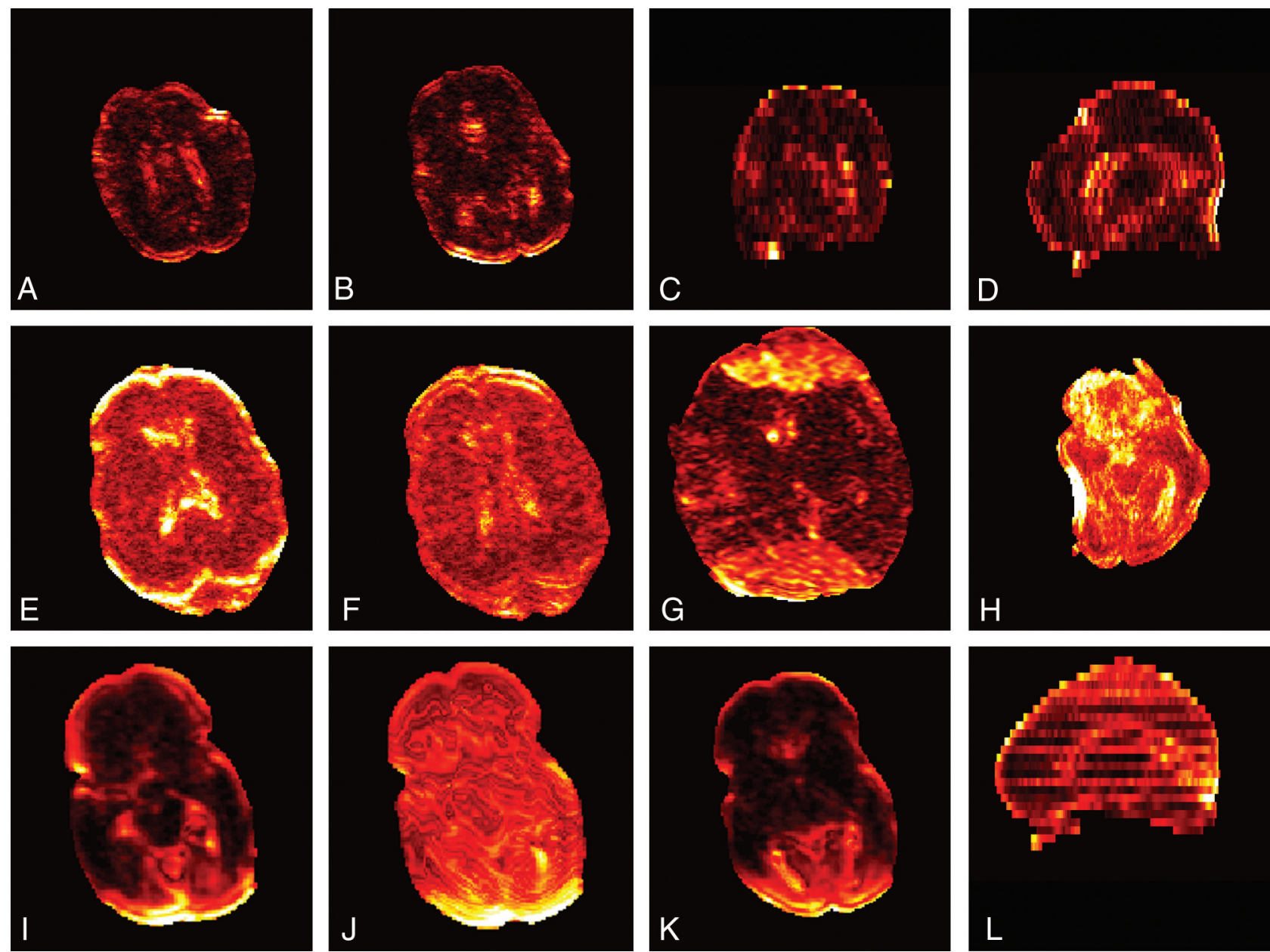

FIG 4. Examples of residuals. $A$ through $D$, Residuals from a subject with good data quality. Higher residuals are present at the border and in the ventricles. $A$ and $B$, Axial images; $C$, coronal view; and $D$, sagittal view. $E$ and $F$, Effects of motion and eddy current correction with higher residuals before correction and lower values after correction; $G$, higher residuals caused by ghosting; $H$, high residuals caused by susceptibility artifacts; I through L, residuals from a subject with gross motion artifacts showing both sections with low (no motion) and high (motion) residuals. The sagittal view (L) shows a pattern with alternating sections of high and lower residuals. Each subject is individually scaled.

istration. A final step is to also correct the diffusion encoding gradients with the same rotational correction as the images. ${ }^{44}$ Neglecting to do so will induce a mismatch between the actual diffusion weighting and the expected diffusion weighting and therefore result in errors in the estimates of the diffusion tensor and the fiber orientation.

It is of paramount importance to ensure that motion correction, which is often necessary in neonatal scanning, does not negatively affect the DTI dataset. Intersection movement is problematic for volume registration, and the $b=0$ volume should be carefully checked on misalignment between sections. Large signal drop-outs are problematic with through-plane motion correction because thereafter, the incorrect signal intensities affect 2 sections, resulting in possible incorrect tensor estimations. Zhou et $\mathrm{al}^{47}$ investigated the use of local texture features to identify and reject outlier images automatically before estimating the diffusion tensor. Their method is fast and removes sections that are corrupted and cannot be used for tensor estimation, resulting in more accurate data. Implementation of fast detection techniques that are based on image characteristics will improve data quality and might offer possibilities to repeat corrupted gradient directions while scanning. ${ }^{53}$

\section{ESTIMATION OF THE TENSOR}

Several tensor estimation methods have been developed to estimate the diffusion tensor. Each of the approaches is based on different principles, and generally speed and accuracy are inversely proportional: methods range from fast but less accurate, to slow but more accurate. ${ }^{9,49,54}$ The main problem in estimating the tensor is the presence of data outliers caused by motion, pulsation, artifacts, noise, and so on. Depending on the method used, these outliers can have a significant impact on the resulting eigenvalues and eigenvectors. The linear leastsquares method is commonly used by vendors and is the default setting for commonly used DTI postprocessing software such as FSL (http://www.fmrib.ox.ac.uk/fsl) ${ }^{55}$; however, this method proved to be the least appropriate to estimate the tensor because the method assumptions are restrictive and physically implausible. ${ }^{56}$ Fig 3 shows the effect of the tensor estimation method for a dataset with few outliers and a dataset corrupted as the result of motion. The difference between the methods is clearly visible, with the ordinary least-squares method providing obviously erroneous results, whereas the weighted least-squares method, which only takes a fraction more computational time, provides more accurate results.

AJNR Am J Neuroradiol 34:1496-505 Aug 2013 www.ajnr.org

1501 

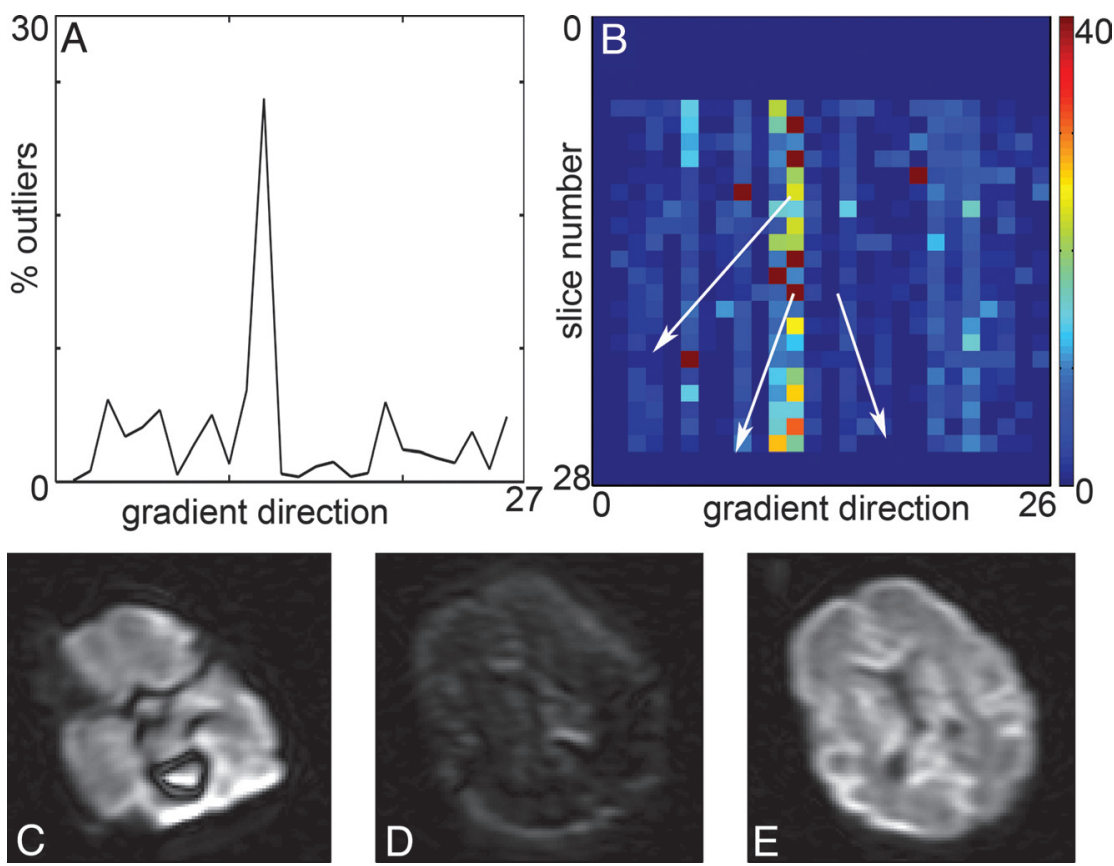

FIG 5. Detection of outliers. A, Percentage of outliers per DWI is a tool to indicate potential problems with the DTI dataset. In this case, DWI 11 and 12 have a high percentage of outliers. B, Percentage outliers per section facilitates easy detection of the problematic sections. During the acquisition of gradient direction 12 , several sections are affected by movement of the infant. $C$ and $D$, Examples of the resulting images; $E$, nonaffected image. Because of the interleaved section acquisition, there is an alternating pattern for the odd and even sections. Scaling is similar for DWI.
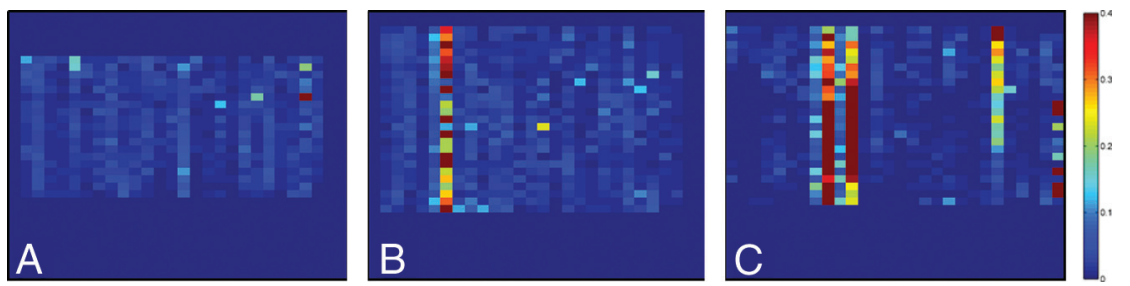

FIG 6. Outlier profile for different datasets. Outliers are only depicted for those sections that contain $>1000$ voxels with signal intensity. $A$, Dataset without gross motion artifacts; $B$, dataset with 1 corrupted gradient direction; $C$, dataset with multiple corrupted gradient directions. Scaling is for $0-40 \%$ outliers.

Therefore, for neonatal DTI data, with its high likelihood of corrupted data, we must use proper tensor estimation procedures to obtain reliable diagnostic images or research data. RESTORE $^{54}$ is a method that detects and removes outlier data in the tensor estimation. This method requires additional gradient encoding directions to eliminate erroneous data. For neonatal DTI measurements, Morris et $\mathrm{al}^{49}$ investigated the use of removing outliers based on the RESTORE algorithm before motion correction because the latter can result in averaging outliers with uncorrupted voxels. With this new method, they found an increased sensitivity to outliers, which is important as outliers correlated strongly with subject movement.

\section{QUALITY ASSURANCE}

It is of paramount importance to ensure that all the steps from data acquisition to data analysis are correct and that the parameter maps are of sufficient quality before analyzing the data and drawing conclusions. Good-quality data are of paramount importance for the neonatal population because the chances of arti- facts and corrupted data are larger than for the average adult population, and artifacts will bias the quantification and increase the variation. Data of sufficient quality will provide quantitative measures with low variation.

Quality assurance begins by carefully checking the $b=0$ images for accuracy and absence of artifacts and intersection movement. Intersection movement causes severe problems with the image registration, resulting in erroneous results; therefore, those image volumes must be excluded from further analysis and hence our previous suggestion to increase the number of $b=0$ volumes. Thereafter, the DWIs are inspected for large signal dropouts, which are easily observed by through-plane projections (Fig 2C). Image misalignments caused by motion or eddy currents can be visualized by a high FA around the rim of the brain or by calculating the standard deviations across the DW images, in which large SD at the rims indicates misregistration. This is also a good method to check whether images were registered accurately or if registration issues persist or even are introduced. Artifacts in the data can also be spotted by locating the areas where FA is $>1$. By definition, this should not be plausible but occurs when the DW signal intensity is larger than the $b=0$ signal intensity.

The tensor estimation model also enables possibilities to spot artifacts by means of the residuals of the tensor fit and the calculation of data outliers. Residuals represent the difference between the fit and the data signal, with high residuals indicating either a poor estimation model or underlying data artifacts. The average residual maps as depicted in Fig 4 are helpful to detect and locate artifacts, assuming that high residuals are caused by artifacts and that good image data result in a homogeneous residuals map. ${ }^{20}$ High residuals are generally found in areas with partial volume effects (rims) and in regions with low signal intensities, ${ }^{20}$ and therefore one should extra carefully interpret results from those regions.

In addition, the residuals are used to detect signal outliers. The location and extent of outliers is a functional tool to investigate the DTI quality. The example in Fig 5 shows that 2 DTI volumes have a high percentage of outliers, which corresponds to corrupted images. We have found that determining the volumes and sections with high percentage outliers is an excellent method to assess data corruption caused by gross motion. ${ }^{50}$ However, labeling a point as an outlier also is dependent on the presence or absence of other (larger) outliers. Indeed, one expects a more or less even 

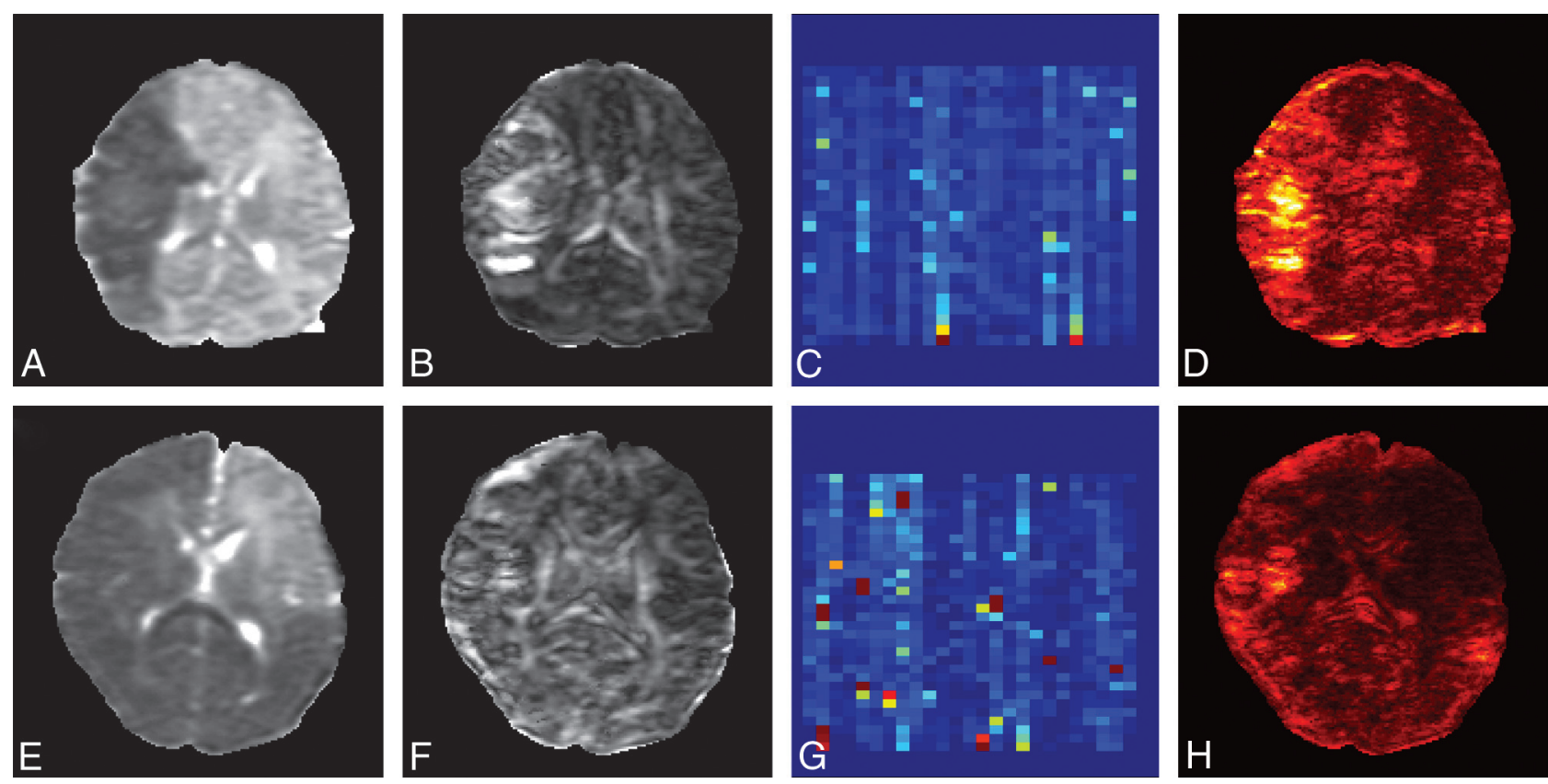

FIG 7. Quality assessment in 2 term-born neonates with brain pathology. The damaged brain areas have much lower $M D(A$ and $E)$ and apparent high FA ( $B$ and $F)$ values. The percentage outliers $(C$ and $G$ ) are low and are distributed uniformly. Because the residuals are also dependent on the underlying $M D$ and FA values, they $\left(D\right.$ and $H$ ) are large in the damaged areas. Scaling: MD, $0-2 \times 10^{-3} \mathrm{~mm}^{2} / \mathrm{s} ; \mathrm{FA}, 0-1 ;$ outliers, $0-40 \%$.

distribution of outliers with high-quality data, whereas corrupted sections cause a significant increase in outliers (Fig 6). Further research is needed to determine the relation between the distribution of outliers and the resulting parameter maps, the needed processing steps, and suitability of a dataset for further analysis.

\section{DATA ANALYSIS}

After the computation of the tensor estimates, the parametric maps can be used for further data analysis to assess the white matter integrity or structure. Commonly used techniques are region of interest selection, voxel-based analysis, tract-based spatial statistics, and fiber tractography. Region of interest selection is used to compare white matter properties within a specific region of the brain. The lower number of comparisons between subjects increases the statistical power, and small differences between subjects may be found. The technique is also independent of individual variations in shape and size of the brain. Major limitations include reliance on the accuracy of the region of interest placements, the likelihood of missing differences in regions that are not included, the time-consuming nature when the regions of interest are manually drawn, and the observer dependency.

Whole-brain analysis can be performed with voxel-based analyses, in which all voxels are analyzed in terms of a tensor estimate. Because of individual variation in size and shape of the brain, all individual brains must first be aligned to a template (normalization) before they can be compared. ${ }^{57,58}$ Normalization is the main challenge in voxel-based analysis because inaccurate normalization results in incorrect comparison of individuals. ${ }^{59}$ This is particularly problematic in patients with cerebral injuries where normalization results in distortion of the brains. TBSS overcomes this limitation by aligning the FA images from multiple subjects to a "mean FA skeleton." With this technique, only voxels that are at the center of tracts common to all the individuals in the population are included. Because voxels with poor alignment are excluded, one of the limitations of this strategy is that it may miss variations in the periphery of white matter tracts. Other limitations and concerns related to TBSS have been addressed by others. ${ }^{60,61}$

Fiber tractography enables delineation and comparison of the orientation and direction of a specific white matter tract between individuals. This method is independent of variation in brain organization. In the deterministic approach, ${ }^{62}$ the algorithm moves in the direction of the principal eigenvector $\left(\varepsilon_{1}\right)$; this is assumed to be parallel to the dominant fiber orientation in each voxel. The main limitation of deterministic tractography is uncertainty about the reliability of a reconstructed trajectory. Probabilistic tractography takes the uncertainty in $\varepsilon_{1}$ into account by propagating a distribution of possible orientations from the seed point. ${ }^{63}$ Although probabilistic tractography seems more reliable, it remains dependent on the placement of the seed points, which is manually done. , $^{3,2,35,64-66}$

Although these analytic measurements provide in vivo information about the orientation, organization, and microstructural properties of the cerebral white matter, DTI analysis remains a proxy technique and not a direct visualization of the cerebral white matter. Moreover, because tensor estimations are subject to error, we emphasize the importance of appropriate acquisition settings with regard to gestational age, a quality check before postprocessing, reliable postprocessing techniques, and good knowledge of neuro anatomy. ${ }^{35}$

\section{DTI QUALITY AND PATHOLOGY}

Investigation of DTI results with pathology brings out the importance of accurate data acquisition, processing, and data interpretation. Fig 7 shows 2 examples of DTI measurements: a patient with a middle cerebral artery infarct and a patient diagnosed with massive cerebral infarction, both scanned within days after the AJNR Am J Neuroradiol 34:1496-505 Aug 2013 www.ajnr.org 
injury. Large portions of the brain are affected, and these consequently have lower MD values as the result of edema. These datasets appear to be of lesser quality in terms of outliers and residuals than the nonpathologic datasets without motion corruption. However, a closer look reveals that the outliers are randomly distributed in time and that the residuals are high in the areas with low MD values. This is expected because the diffusivities in the pathologic areas are lower and therefore the b-value might not be optimal to accurately determine MD and FA values in these areas. Although data quality assurance is not directly a vital issue for clinical practice in which the main goal is to visualize the areas with altered MD and FA values, it is imperative for the analysis of the DTI values to exclude any bias in DTI indices.

\section{CONCLUSIONS}

High-quality, quantitative data are essential to ensure reliable and meaningful imaging findings. Both data quality and analysis will improve with the use of protocols and image-processing tools specifically designed for neonatal imaging, seeing that tissue composition and occurrence of artifacts in neonates are significantly different from those in the adult population. Optimized acquisition and targeted processing will increase the diagnostic and prognostic value of the scans but will also considerably improve intervention or outcome studies because a reduced data spread will lead to stronger correlations, will require fewer subjects, and will allow earlier determination of valuable intervention methods.

\section{REFERENCES}

1. Counsell SJ, Rutherford MA, Cowan FM, et al. Magnetic resonance imaging of preterm brain injury. Arch Dis Child Fetal Neonatal Ed 2003;88:F269-74

2. Huppi PS, Barnes PD. Magnetic resonance techniques in the evaluation of the newborn brain. Clin Perinatol 1997;24:693-723

3. Ment LR, Hirtz D, Huppi PS. Imaging biomarkers of outcome in the developing preterm brain. Lancet Neurol 2009;8:1042-55

4. Lequin MH, Dudink J, Tong KA, et al. Magnetic resonance imaging in neonatal stroke. Semin Fetal Neonatal Med 2009;14:299-310

5. Skiold B, Horsch S, Hallberg B, et al. White matter changes in extremely preterm infants, a population-based diffusion tensor imaging study. Acta Paediatr 2010;99:842-49

6. Gimenez M, Miranda MJ, Born AP, et al. Accelerated cerebral white matter development in preterm infants: a voxel-based morphometry study with diffusion tensor MR imaging. Neuroimage 2008;41:728-34

7. Basser PJ, Mattiello J, LeBihan D. Estimation of the effective selfdiffusion tensor from the NMR spin echo. J Magn Reson 1994;103:247-54

8. Pierpaoli C, Jezzard P, Basser PJ, et al. Diffusion tensor MR imaging of the human brain. Radiology 1996;201:637-48

9. Le Bihan D, van Zijl P. From the diffusion coefficient to the diffusion tensor. NMR Biomed 2002;15:431-34

10. Mori S, van Zijl PC. Fiber tracking: principles and strategies: a technical review. NMR Biomed 2002;15:468-80

11. Jones DK, Leemans A. Diffusion tensor imaging. Methods Mol Biol 2011;711:127-44

12. Dudink J, Kerr JL, Paterson K, et al. Connecting the developing preterm brain. Early Hum Dev 2008;84:777-82

13. Anjari M, Srinivasan L, Allsop JM, et al. Diffusion tensor imaging with tract-based spatial statistics reveals local white matter abnormalities in preterm infants. Neuroimage 2007;35:1021-27

14. Bassi L, Chew A, Merchant N, et al. Diffusion tensor imaging in preterm infants with punctate white matter lesions. Pediatr Res 2011;69:561-66
15. van Kooij BJ, van Pul C, Benders MJ, et al. Fiber tracking at term displays gender differences regarding cognitive and motor outcome at 2 years of age in preterm infants. Pediatr Res 2011;70:626-32

16. Bassi L, Ricci D, Volzone A, et al. Probabilistic diffusion tractography of the optic radiations and visual function in preterm infants at term equivalent age. Brain 2008;131:573-82

17. Drobyshevsky A, Bregman J, Storey P, et al. Serial diffusion tensor imaging detects white matter changes that correlate with motor outcome in premature infants. Dev Neurosci 2007;29:289-301

18. van Kooij BJ, de Vries LS, Ball G, et al. Neonatal tract-based spatial statistics findings and outcome in preterm infants. AJNR Am J Neuroradiol 2012;33:188-94

19. Jones DK, Cercignani M. Twenty-five pitfalls in the analysis of diffusion MRI data. NMR Biomed 2010;23:803-20

20. Tournier JD, Mori S, Leemans A. Diffusion tensor imaging and beyond. Magn Reson Med 2011;65:1532-56

21. Johansen-Berg H, Behrens TE, eds. Diffusion MRI: from quantitative measurement to in-vivo neuroanatomy. 2009. Waltham, Massachusetts: Academic Press, Elsevier.

22. Jones DK, Horsfield MA, Simmons A. Optimal strategies for measuring diffusion in anisotropic systems by magnetic resonance imaging. Magn Reson Med 1999;42:515-25

23. Battin M, Maalouf EF, Counsell S, et al. Physiological stability of preterm infants during magnetic resonance imaging. Early Hum Dev 1998;52:101-10

24. Benavente-Fernandez I, Lubian-Lopez PS, Zuazo-Ojeda MA, et al. Safety of magnetic resonance imaging in preterm infants. Acta Paediatr 2010;99:850-53

25. Merchant N, Groves A, Larkman DJ, et al. A patient care system for early 3.0 Tesla magnetic resonance imaging of very low birth weight infants. Early Hum Dev 2009;85:779-83

26. Plaisier A, Raets MM, van der Starre C, et al. Safety of routine early MRI in preterm infants. Pediatr Radiol 2012;42:1205-11

27. Keil B, Alagappan V, Mareyam A, et al. Size-optimized 32-channel brain arrays for $3 \mathrm{~T}$ pediatric imaging. Magn Reson Med 2011;66:1777-87

28. Legendre V, Burtner PA, Martinez KL, et al. The evolving practice of developmental care in the neonatal unit: a systematic review. Phys Occup Ther Pediatr 2011;31:315-38

29. Neubauer V, Griesmaier E, Baumgartner K, et al. Feasibility of cerebral MRI in non-sedated preterm-born infants at term-equivalent age: report of a single centre. Acta Paediatr 2011;100:1544-47

30. Nordell A, Lundh M, Horsch S, et al. The acoustic hood: a patientindependent device improving acoustic noise protection during neonatal magnetic resonance imaging. Acta Paediatr 2009;98: $1278-83$

31. Holland BA, Haas DK, Norman D, et al. MRI of normal brain maturation. AJNR Am J Neuroradiol 1986;7:201-08

32. Counsell SJ, Kennea NL, Herlihy AH, et al. T2 relaxation values in the developing preterm brain. AJNR Am J Neuroradiol 2003;24:1654-60

33. Kumar R, Delshad S, Macey PM, et al. Development of T2-relaxation values in regional brain sites during adolescence. Magn Reson Imaging 2011;29:185-93

34. Vos SB, Jones DK, Viergever MA, et al. Partial volume effect as a hidden covariate in DTI analyses. Neuroimage 2011;55:1566-76

35. Mukherjee P, Chung SW, Berman JI, et al. Diffusion tensor MR imaging and fiber tractography: technical considerations. AJNR AmJ Neuroradiol 2008;29:843-52

36. Reese TG, Heid O, Weisskoff RM, et al. Reduction of eddy-currentinduced distortion in diffusion MRI using a twice-refocused spin echo. Magn Reson Med 2003;49:177-82

37. Pierpaoli C, Basser PJ. Toward a quantitative assessment of diffusion anisotropy. Magn Reson Med 1996;36:893-906

38. Damon BM. Effects of image noise in muscle diffusion tensor (DT)MRI assessed using numerical simulations. Magn Reson Med 2008;60:934-44

39. Chenevert TL, Galban CJ, Ivancevic MK, et al. Diffusion coefficient 
measurement using a temperature-controlled fluid for quality control in multicenter studies. J Magn Reson Imaging 2011;34:983-87

40. Bammer R, Schoenberg SO. Current concepts and advances in clinical parallel magnetic resonance imaging. Top Magn Reson Imaging 2004;15:129-58

41. Jones DK. The effect of gradient sampling schemes on measures derived from diffusion tensor MRI: a Monte Carlo study. Magn Reson Med 2004;51:807-15

42. Cook PA, Symms M, Boulby PA, et al. Optimal acquisition orders of diffusion-weighted MRI measurements. J Magn Reson Imaging 2007;25:1051-58

43. Dubois J, Poupon C, Lethimonnier F, et al. Optimized diffusion gradient orientation schemes for corrupted clinical DTI data sets. MAGMA 2006;19:134-43

44. Leemans A, Jones DK. The B-matrix must be rotated when correcting for subject motion in DTI data. Magn Reson Med 2009;61: $1336-49$

45. Ling J, Merideth F, Caprihan A, et al. Head injury or head motion? Assessment and quantification of motion artifacts in diffusion tensor imaging studies. Hum Brain Mapp 2012;33:50-62

46. Pierpaoli C, Marenco S, Rohde G, et al. Analyzing the contribution of cardiac pulsation to the variability of quantities derived from the diffusion tensor. ISMRM Toronto, Canada, July 10-16, 2003:70

47. Zhou Z, Liu W, Cui J, et al. Automated artifact detection and removal for improved tensor estimation in motion-corrupted DTI data sets using the combination of local binary patterns and 2D partial least squares. Magn Reson Imaging 2011;29:230-42

48. Dudink J, Lequin M, van Pul C, et al. Fractional anisotropy in white matter tracts of very-low-birth-weight infants. Pediatr Radiol 2007;37:1216-23

49. Morris D, Nossin-Manor R, Taylor MJ, et al. Preterm neonatal diffusion processing using detection and replacement of outliers prior to resampling. Magn Reson Med 2011;66:92-101

50. Heemskerk AM, Plaisier A, Reiss I, et al. DTI in neonates: data corruption due to motion. ISMRM Melbourne, Australia, May 5-11, 2012

51. Tofts PS, Lloyd D, Clark CA, et al. Test liquids for quantitative MRI measurements of self-diffusion coefficient in vivo. Magn Reson Med 2000;43:368-74

52. Kozak LR, Bango M, Szabo M, et al. Using diffusion MRI for measuring the temperature of cerebrospinal fluid within the lateral ventricles. Acta Paediatr 2010;99:237-43

53. Aksoy M, Forman C, Straka M, et al. Real-time optical motion correction for diffusion tensor imaging. Magn Reson Med 2011; 66:366-78

54. Chang LC, Jones DK, Pierpaoli C. RESTORE: robust estimation of tensors by outlier rejection. Magn Reson Med 2005;53:1088-95

55. Smith SM, Jenkinson M, Woolrich MW, et al. Advances in functional and structural MR image analysis and implementation as FSL. Neuroimage 2004;23(Suppl 1):S208-19

56. Koay CG, Chang LC, Carew JD, et al. A unifying theoretical and algorithmic framework for least squares methods of estimation in diffusion tensor imaging. J Magn Reson 2006;182:115-25

57. Van Hecke W, Leemans A, D'Agostino E, et al. Nonrigid coregistration of diffusion tensor images using a viscous fluid model and mutual information. IEEE Trans Med Imaging 2007;26:1598-612

58. Van Hecke W, Sijbers J, D'Agostino E, et al. On the construction of an inter-subject diffusion tensor magnetic resonance atlas of the healthy human brain. Neuroimage 2008;43:69-80

59. Van Hecke W, Leemans A, Sage CA, et al. The effect of template selection on diffusion tensor voxel-based analysis results. Neuroimage 2011;55:566-73
60. Edden RA, Jones DK. Spatial and orientational heterogeneity in the statistical sensitivity of skeleton-based analyses of diffusion tensor MR imaging data. J Neurosci Methods 2011;201:213-19

61. Van Hecke W, Leemans A, De Backer S, et al. Comparing isotropic and anisotropic smoothing for voxel-based DTI analyses: a simulation study. Hum Brain Mapp 2010;31:98-114

62. Basser PJ, Pajevic S, Pierpaoli C, et al. In vivo fiber tractography using DT-MRI data. Magn Reson Med 2000;44:625-32

63. Jones DK. Tractography gone wild: probabilistic fibre tracking using the wild bootstrap with diffusion tensor MRI. IEEE Trans Med Imaging 2008;27:1268-74

64. Feldman HM, Yeatman JD, Lee ES, et al. Diffusion tensor imaging: a review for pediatric researchers and clinicians. J Dev Behav Pediatr 2010;31:346-56

65. Jones DK. Studying connections in the living human brain with diffusion MRI. Cortex 2008;44:936-52

66. Huppi PS, Dubois J. Diffusion tensor imaging of brain development. Semin Fetal Neonatal Med 2006;11:489-97

67. van Pul C, van Kooij BJ, de Vries LS, et al. Quantitative fiber tracking in the corpus callosum and internal capsule reveals microstructural abnormalities in preterm infants at term-equivalent age. AJNR Am J Neuroradiol 2012;33:678-84

68. Wintermark P, Hansen A, Gregas MC, et al. Brain perfusion in asphyxiated newborns treated with therapeutic hypothermia. AJNR Am J Neuroradiol 2011;32:2023-29

69. Arrigoni F, Parazzini C, Righini A, et al. Deep medullary vein involvement in neonates with brain damage: an MR imaging study. AJNR Am J Neuroradiol 2011;32:2030-36

70. Oishi K, Mori S, Donohue PK, et al. Multi-contrast human neonatal brain atlas: application to normal neonate development analysis. Neuroimage 2011;56:8-20

71. Wang Y, Gupta A, Liu Z, et al. DTI registration in atlas based fiber analysis of infantile Krabbe disease. Neuroimage 2011;55:1577-86

72. Hasegawa T, Yamada K, Morimoto M, et al. Development of corpus callosum in preterm infants is affected by the prematurity: in vivo assessment of diffusion tensor imaging at term-equivalent age. $P e-$ diatr Res 2011;69:249-54

73. de Bruine FT, van Wezel-Meijler G, Leijser LM, et al. Tractography of developing white matter of the internal capsule and corpus callosum in very preterm infants. Eur Radiol 2011;21:538-47

74. Righini A, Doneda C, Parazzini C, et al. Diffusion tensor imaging of early changes in corpus callosum after acute cerebral hemisphere lesions in newborns. Neuroradiology 2010;52:1025-35

75. Bonifacio SL, Glass HC, Chau V, et al. Extreme premature birth is not associated with impaired development of brain microstructure. J Pediatr 2010;157:726-32 el

76. Gilmore JH, Kang C, Evans DD, et al. Prenatal and neonatal brain structure and white matter maturation in children at high risk for schizophrenia. Am J Psychiatry 2010;167:1083-91

77. Dudink J, Buijs J, Govaert P, et al. Diffusion tensor imaging of the cortical plate and subplate in very-low-birth-weight infants. Pediatr Radiol 2010;40:1397-404

78. Liu Y, Baleriaux D, Kavec M, et al. Structural asymmetries in motor and language networks in a population of healthy preterm neonates at term equivalent age: a diffusion tensor imaging and probabilistic tractography study. Neuroimage 2010;51:783-88

79. Malik GK, Trivedi R, Gupta A, et al. Quantitative DTI assessment of periventricular white matter changes in neonatal meningitis. Brain Dev 2008;30:334-41

80. Rose SE, Hatzigeorgiou X, Strudwick MW, et al. Altered white matter diffusion anisotropy in normal and preterm infants at term-equivalent age. Magn Reson Med 2008;60:761-67 